\title{
TP83 - MicroCT Data Acquisition, Reconstruction and Analysis Using the ISA MicroCT System
}

\author{
William D. Brown \\ Lawrence Livermore National Laboratory \\ Livermore, CA 94551 \\ Work performed for the \\ Science \& Technology Directorate of the \\ Department of Homeland Security \\ Statement of Work \\ PR RSEN-08-00066 \\ January 30, 2014 \\ Version 1.1 \\ LLNL-TR-649192 \\ IM\# 768139
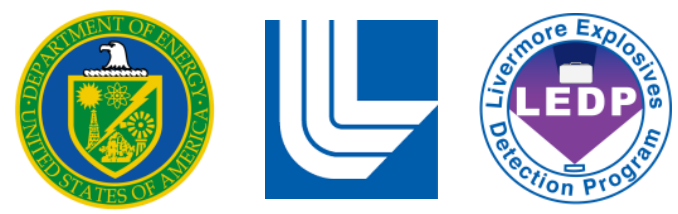
This document was prepared as an account of work sponsored by an agency of the United States government. Neither the United States government nor Lawrence Livermore National Security, LLC, nor any of their employees makes any warranty, expressed or implied, or assumes any legal liability or responsibility for the accuracy, completeness, or usefulness of any information, apparatus, product, or process disclosed, or represents that its use would not infringe privately owned rights. Reference herein to any specific commercial product, process, or service by trade name, trademark, manufacturer, or otherwise does not necessarily constitute or imply its endorsement, recommendation, or favoring by the United States government or Lawrence Livermore National Security, LLC. The views and opinions of authors expressed herein do not necessarily state or reflect those of the United States government or Lawrence Livermore National Security, LLC, and shall not be used for advertising or product endorsement purposes.

This work performed under the auspices of the U.S. Department of Energy by Lawrence Livermore National Laboratory under Contract DE-AC52-07NA27344. 


\section{TP83 - MicroCT Data Acquisition, Reconstruction and Analysis Using the ISA MicroCT System}

\section{Objectives}

The objective of this test plan is to acquire MicroCT small scale data $(60 \mathrm{~mL})$ samples using the ISA MicroCT system. The data will be used to acquire x-ray properties of the sample.

\section{Summary of Approach}

A specimen of interest will be housed inside a 60-mL Low Density Polyethylene (LDPE) container. The sample will be placed on the MicroCT carousel and $3 \mathrm{CT}$ data scans will be acquired. The first scan (exp1) uses a $160 \mathrm{kV}$ bremsstrahlung X-ray source with Aluminum and Copper filtering configured as a fan beam. The second scan (exp2) uses a $100 \mathrm{kV}$ x-ray source with Aluminum filtering with a fan beam configuration. The third scan (exp1_Open) is identical to exp1 but the configuration is a cone beam. Each data set will be reconstructed using LLNL developed software and LLNL developed reconstruction scripts.

System performance will be monitored through analysis of the carousel references and energy dependent copper strip attenuation values. If the references and copper strip are within Quality Assurance (QA) bounds, the scan will be considered acceptable. If they are outside the bounds, the scans will be considered unacceptable and a new data set will be generated.

The reconstructed sample images will be analyzed using LLNL developed segmentation and analysis code. The output of the analysis is an Excel spreadsheet with x-ray properties of the sample.

\section{Materials}
a) ISA MicroCT System [Brown]
b) Specimen
c) Nalgene $60-\mathrm{mL}_{\text {bottles }}{ }^{1}$

\section{MicroCT System Alignment}

For proper reconstruction of the CT data, the MicroCT system must be aligned and the geometry of the system be defined. The system will be aligned by following the alignment procedure [Divin]. An abbreviated alignment acquisition checklist can be found in reference [Pincus]. It is recommended the system be re-aligned whenever there has been preventative maintenance performed on the system, when any of the system components have been replaced, or if there is a potential the x-ray tube head and/or detector may have been disturbed. In addition, it is recommended the alignment procedure be executed and the results recorded on a monthly basis. The monthly recordings can be used to track the stability of the alignment.

\footnotetext{
${ }^{1}$ www.Fishersci.com, Part Number 02-896-1B, 60 mL capacity
} 


\section{Detector Calibration}

The flat panel detector requires a calibration to be performed to allow uniformity and linearity of the panel during data acquisition. The operator will acquire the calibration files by following the calibration procedure checklist [Pincus]. The detector shall be calibrated at the energies used for data acquisition and with the slit collimator removed. New calibrations should be acquired on a monthly basis or whenever there has been a realignment of the MicroCt system.

\section{MicroCT Data Acquisition Procedure}

Three CT Scan will be performed for each sample. The three scans are $160 \mathrm{kV}, 2$ slit with Aluminum and Copper filtering (exp1), $100 \mathrm{kV}, 2$ slit with Aluminum filtering (exp2) and $160 \mathrm{kV}, 1$ slit, with Aluminum and Copper filtering (exp1_Open). Appendix A contains the three techniques for each scan. These techniques contain the scanning parameters. The operator shall follow the data acquisition checklist that provides stepby-step instructions for acquiring each of the 3 data acquisition scans [Pincus].

The three scans should be acquired on the same day and it is recommended the scan order should be:

$$
\begin{aligned}
& \text { Exp1 - } 160 \mathrm{kV} \mathrm{Al/Cu} \mathrm{Filter}-2 \text { Slits } \\
& \text { Exp2-100 kV Al Filter }-2 \text { Slits } \\
& \text { Exp1_Open - } 160 \mathrm{kV} \mathrm{Al/Cu} \mathrm{Filter} \mathrm{-} 1 \text { Slit }
\end{aligned}
$$

Use the applicable technique sheet to setup the scan parameters and the checklist to acquire each data scan.

\section{Copper Strip QA Check}

To verify the $\mathrm{x}$-ray machine is generating a repeatable spectrum, LLNL developed software to monitor the copper strip. The name of the copper strip analysis software is Cu Strip GUI. To run the Cu Strip GUI refer to the Cu Strip GUI manual [Seetho1]. After the software has completed, the resultant values of the mean lower slit and the ratio of the mean upper slit to mean lower slit will be compared to the copper strip criteria. Appendix B contains the copper strip QA Boundaries and criteria. If the scan fails the criteria requirement, it may be an indication the system has changed and may warrant rescanning the sample. If the copper strip results pass the criteria continue to Section 8 .

\section{Reconstruction of MicroCT data and Qualitative Review}

The MicroCT data will be reconstructed using the LLNL developed software IMGREC [Schneberk]. The reconstructions will be performed on the ISA System reconstruction and analysis computer. Appendix $\mathrm{C}$ contains example scripts to execute the reconstruction from IMGREC. The actual scripts may change whenever the system has undergone preventative maintenance or there has been a change in the system hardware. The following tasks will be used to reconstruct the exp1 data:

a. Place the exp1 reconstruction script in the same directory as the exp1 data.

b. Double click on the IMGREC Icon to start the program. 
c. Using the Set Current Dir window, set the directory to the exp1 data set.

d. Click File $I O=>$ ReadComFile and click on the exp1 script file.

e. In the Command File Processing window, click the Do-Com-File button to start the reconstruction.

f. When the reconstruction is complete the Current Command in the Command File Processing Window will state "Reconstructions Complete".

Repeat steps a. thru f. for the exp2 and exp1_Open data sets. After the reconstructions are complete, the data will be qualitatively reviewed to check for centering artifacts or a bad beam hardening correction in exp2. Review all reconstructed images in each data set. If the data shows horseshoe artifacts the centering of the data set will be changed, the scripts modified to include the new centering value and the reconstruction re-run with the modified scripts. LLNL will be notified if the scripts change and a copy of the modified scripts will be sent to LLNL. Refer to the IMGREC manual [Schneberk] to change the centering parameter. For the exp2 data set, the aluminum reference will be checked for beam hardening correction [Schneberk]. If there is evidence of cupping in the aluminum reference, LLNL should be consulted for correction.

\section{MicroCT Reference Analysis}

The MicroCT exp1 and exp2 data set references will be analyzed using the LLNL developed software Automated MicroCT Analysis GUI (AMAG). To run AMAG refer to the AMAG Standard Operating Procedure (SOP) [Seetho2]. After the software has completed, open the resultant spreadsheet and compare each reference value to the reference QA Boundaries and reference criteria. The reference boundaries and reference criteria can be found in Appendix B. If the references meet the criterion, the scans are considered acceptable. If the criterion is not met, it may be an indication the system has changed and may warrant rescanning the sample. 
References

Brown Brown, W.D., Documentation of the ISA Micro Computed Tomography System, Lawrence Livermore National Laboratory, LLNL-TR-XXXXXX, December 18, 2013

Divin Divin, C., MicroCT: Procedure for Alignment, Lawrence Livermore National Laboratory, LEDP-MCT-SOP-010, Lawrence Livermore National Laboratory, December 11, 2012

Pincus Pincus, C, MicroCT Data Acquisition Checklist, Lawrence Livermore National Laboratory, LLNL-TR-XXXXXX, May 1, 2013

Seetho1 Seetho, I., J. S. Kallman, W. T. White, H.E. Martz, MicroCT: X-Ray Radiograph Qualtity Assurance Through the Analysis of Copper Strip Data Using the Matlab CuStrip Analysis GUI, LLNL-TR-588852, October, 8, 2012

Schneberk Schneberk, D., Imgrec User Guide: CT Pre-processing, Reconstruction, and Image Processing and Image Inspection Tools, Version 10, Lawrence Livermore National Laboratory, March 1, 2010

Seetho2 Seetho, Isaac, MicroCT: Automated Analysis of CT Reconstructed Data of Home Made Explosive Materials Using the Matlab MicroCT Analysis GUI, Lawrence Livermore National Lab, LLNL-TR-503291, October 6, 2011 


\section{Appendix A}

\section{Data Acquisition Technique Sheets}

\begin{tabular}{|c|c|}
\hline Title & 160 kV 2 Slit Al/Cu Filter - exp1 \\
\hline \multicolumn{2}{|l|}{ System Information } \\
\hline Data Acquistion Date & Monday, January 13, 2014 \\
\hline System & ISA 450kV \\
\hline Archive Directory & C:IISA System\CT Data\2014_Jan_SAT \\
\hline \multicolumn{2}{|l|}{ Source } \\
\hline X-ray Source & Yxlon 450kV D11 \\
\hline Effective Spot Size (mm) & Small 0.4 \\
\hline Energy (kV) & 160 \\
\hline Tube Current (mA) & 4.35 \\
\hline Geometric Unsharpness (mm) & 0.112 \\
\hline Filter (Type/Thickness mm) & $\mathrm{Al} / 2.0 \mathrm{Cu} / 2.0$ \\
\hline \multicolumn{2}{|l|}{ Detector } \\
\hline Detector Type & Amorphous Silicon Perkin Elmer \\
\hline Source-Detector-Distance (SDD) (mm) & 1413.4 \\
\hline Source-Object-Distance (SOD) (mm) & 1103.8 \\
\hline Object-Detector-Distance (ODD) (mm) & 309.6 \\
\hline X-Offset (Pixels) & 309 \\
\hline X-Size (Pixels) & 1694 \\
\hline Y-Offset (Pixels) & 995 \\
\hline Y-Size (Pixels) & 255 \\
\hline Magnification & 1.280 \\
\hline Frame Average(s) & 6 \\
\hline Integration Knob-Thales Only & $\mathrm{N} / \mathrm{A}$ \\
\hline Integration Time per Frame(sec) & 1.15 \\
\hline Raw Pixel Size $(\mathrm{mm})$ & 0.2 \\
\hline Global Resampling & 1 \\
\hline Effective Pixel size at Detector (mm) & 0.2 \\
\hline Effective Pixel size at Object (mm) & 0.156 \\
\hline \multicolumn{2}{|l|}{ CT Parameters } \\
\hline Numbers of Views & 401 \\
\hline Angular Range & 200.5 \\
\hline Angular Step (Degrees) & 0.5 \\
\hline Estimated PxCenterFull Panel (Pixel) & 1023.1 \\
\hline Estimated PzCenterFull Panel (Pixel) & 1027.8 \\
\hline
\end{tabular}




\begin{tabular}{|l|l|}
\hline Title & $100 \mathrm{kV} 2$ Slit Al Filter - exp2 \\
\hline & \\
\hline System Information & Monday, January 13, 2014 \\
\hline Data Acquistion Date & ISA 450kV \\
\hline System & C:ISA System ICT Data\2014_Jan_SAT \\
\hline Archive Directory & \\
\hline & \\
\hline Source & Yxlon 450kV D11 \\
\hline X-ray Source & Small 0.4 \\
\hline Effective Spot Size (mm) & 100 \\
\hline Energy (kV) & 7 \\
\hline Tube Current (mA) & 0.112 \\
\hline Geometric Unsharpness (mm) & Al/2.0 \\
\hline Filter (Type/Thickness mm) & \\
\hline & \\
\hline Detector & Amorphous Silicon Perkin Elmer \\
\hline Detector Type & 1413.4 \\
\hline Source-Detector-Distance (SDD) (mm) & 1103.8 \\
\hline Source-Object-Distance (SOD) (mm) & 309.6 \\
\hline Object-Detector-Distance (ODD) (mm) & 309 \\
\hline X-Offset (Pixels) & 1694 \\
\hline X-Size (Pixels) & 995 \\
\hline Y-Offset (Pixels) & 255 \\
\hline Y-Size (Pixels) & 1.280 \\
\hline Magnification & 6 \\
\hline Frame Average(s) & N/A \\
\hline Integration Knob-Thales Only & 0.267 \\
\hline Integration Time per Frame(sec) & 0.2 \\
\hline Raw Pixel Size (mm) & 1 \\
\hline Global Resampling & 0.2 \\
\hline Effective Pixel size at Detector (mm) & 0.156 \\
\hline Effective Pixel size at Object (mm) & \\
\hline & \\
\hline CT Parameters & 401 \\
\hline Numbers of Views & 200.5 \\
\hline Angular Range & 0.5 \\
\hline Angular Step (Degrees) & 1023.1 \\
\hline Estimated PxCenterFull Panel (Pixel) & 1027.8 \\
\hline Estimated PzCenterFull Panel (Pixel) & \\
\hline
\end{tabular}




\begin{tabular}{|l|l|}
\hline Title & $160 \mathrm{kV}$ 1Slit Al/Cu Filter - exp1_Open \\
\hline System Information & \\
\hline Data Acquistion Date & Monday, January 13, 2014 \\
\hline System & ISA 450kV \\
\hline Archive Directory & C:ISA System ICT Data/2014_Jan_SAT \\
\hline & \\
\hline Source & Yxlon 450kV D11 \\
\hline X-ray Source & Small 0.4 \\
\hline Effective Spot Size (mm) & 160 \\
\hline Energy (kV) & 4.35 \\
\hline Tube Curren t (mA) & 0.112 \\
\hline Geometric Unsharpness (mm) & Al/2.0 Cu/2.0 \\
\hline Filter (Type/Thickness mm) & \\
\hline & \\
\hline Detector & Amorphous Silicon Perkin Elmer \\
\hline Detector Type & 1413.4 \\
\hline Source-Detector-Distance (SDD) (mm) & 1103.8 \\
\hline Source-Object-Distance (SOD) (mm) & 309.6 \\
\hline Object-Detector-Distance (ODD) (mm) & 309 \\
\hline X-Offset (Pixels) & 1694 \\
\hline X-Size (Pixels) & 495 \\
\hline Y-Offset (Pixels) & 755 \\
\hline Y-Size (Pixels) & 1.280 \\
\hline Magnification & 6 \\
\hline Frame Average(s) & N/A \\
\hline Integration Knob-Thales Only & 0.267 \\
\hline Integration Time per Projection(sec) & 0.2 \\
\hline Raw Pixel Size (mm) & 1 \\
\hline Global Resampling & 0.2 \\
\hline Effective Pixel size at Detector (mm) & 0.156 \\
\hline Effective Pixel size at Object (mm) & \\
\hline & 401 \\
\hline CT Parameters & 200.5 \\
\hline Numbers of Views & 0.5 \\
\hline Angular Range & 1023.1 \\
\hline Angular Step (Degrees) & 1027.8 \\
\hline Estimated PxCenterFull Panel (Pixel) & \\
\hline Estimated PzCenterFull Panel (Pixel) & \\
\hline
\end{tabular}




\section{Appendix B}

\section{Copper Strip QA Boundaries and Criteria}

\begin{tabular}{|c|c|c|c|c|}
\cline { 2 - 5 } \multicolumn{1}{c|}{} & \multicolumn{2}{|c|}{ Mean Lower Slit } & \multicolumn{2}{c|}{ Lower Slit/Upper Slit } \\
\hline & Attenuation & \multicolumn{2}{c|}{ Ratio } \\
\hline $160 \mathrm{kV} \mathrm{AlCu}$ & 0.6833 & 0.6919 & 1.0016 & 1.0117 \\
\hline $100 \mathrm{kV} \mathrm{Al}$ & 1.9725 & 1.9926 & 1.0062 & 1.0086 \\
\hline
\end{tabular}

MicroCT Copper Strip QA Boundaries

\begin{tabular}{|l|c|c|c|c|}
\hline Material & $\begin{array}{c}160 \mathrm{kV} \\
\text { Mean Min }\end{array}$ & $\begin{array}{c}160 \mathrm{kV} \\
\text { Mean Max }\end{array}$ & $\begin{array}{c}\text { Ratio } \\
\text { Min }\end{array}$ & $\begin{array}{c}\text { Ratio } \\
\text { Max }\end{array}$ \\
\hline Silicon & 2569.04 & 2605.76 & 2.082 & 2.101 \\
\hline Delrin & 1321.65 & 1336.65 & 1.328 & 1.342 \\
\hline Graphite & 1568.26 & 1588.85 & 1.252 & 1.263 \\
\hline Magnesium & 1807.78 & 1822.03 & 1.911 & 1.920 \\
\hline Water & 999.00 & 1001.00 & 1.372 & 1.388 \\
\hline Teflon & 1896.67 & 1937.53 & 1.468 & 1.484 \\
\hline
\end{tabular}

MicroCT Reference QA Bounds 


\section{Appendix C \\ Reconstruction Scripts}

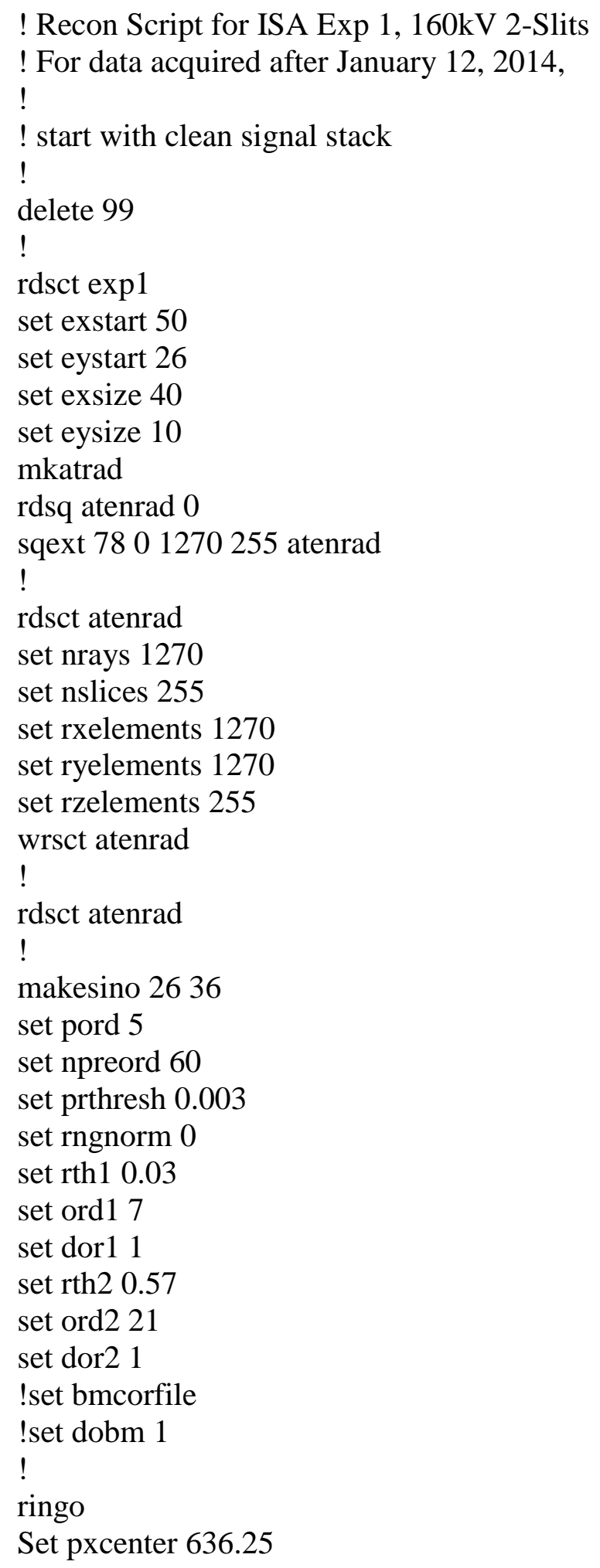




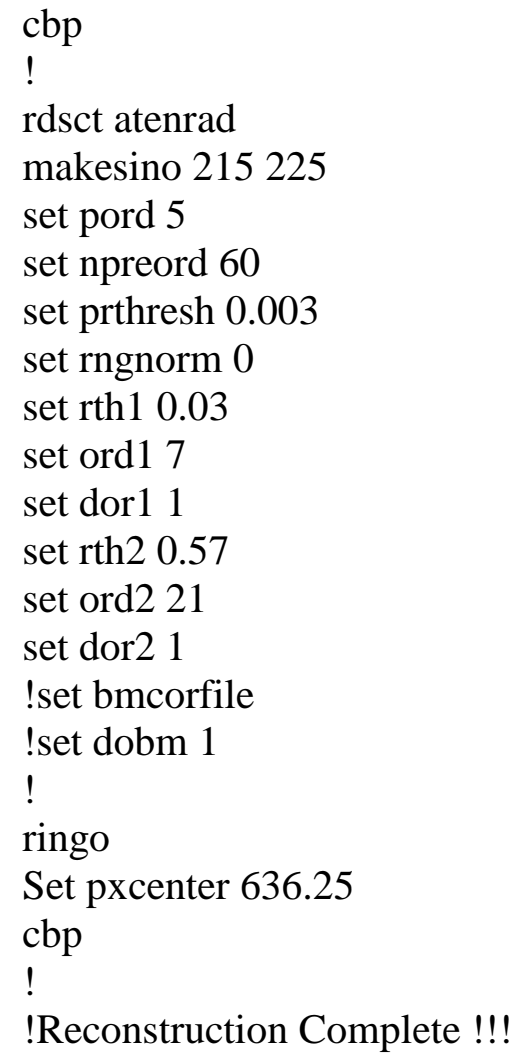




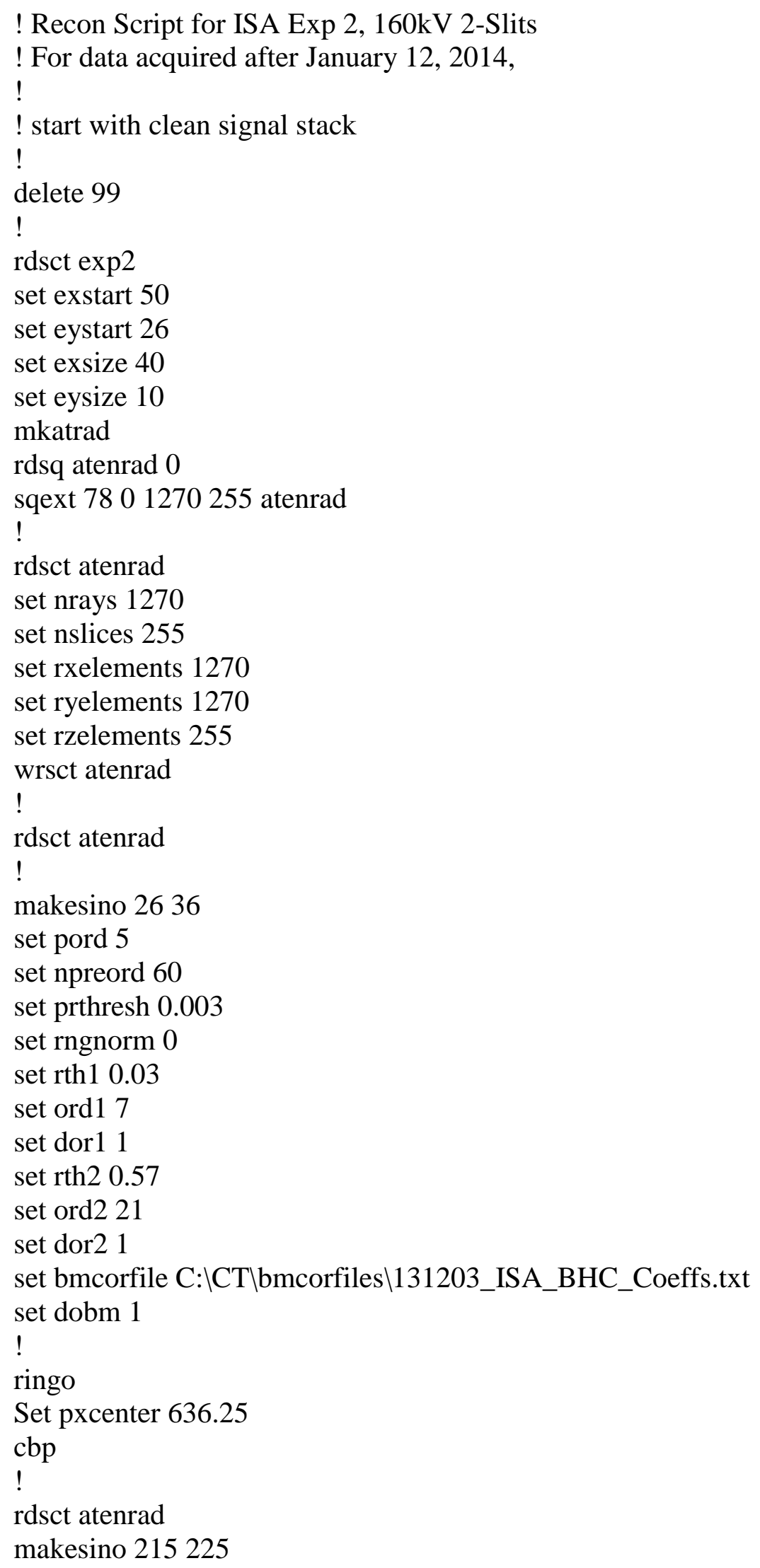


set pord 5

set npreord 60

set prthresh 0.003

set rngnorm 0

set rth1 0.03

set ord 17

set dor 11

set rth2 0.57

set ord 221

set dor2 1

set bmcorfile C:ICT \bmcorfiles\131203_ISA_BHC_Coeffs.txt

set dobm 1

!

ringo

Set pxcenter 636.25

$\operatorname{cbp}$

!

!Reconstruction Complete !!! 


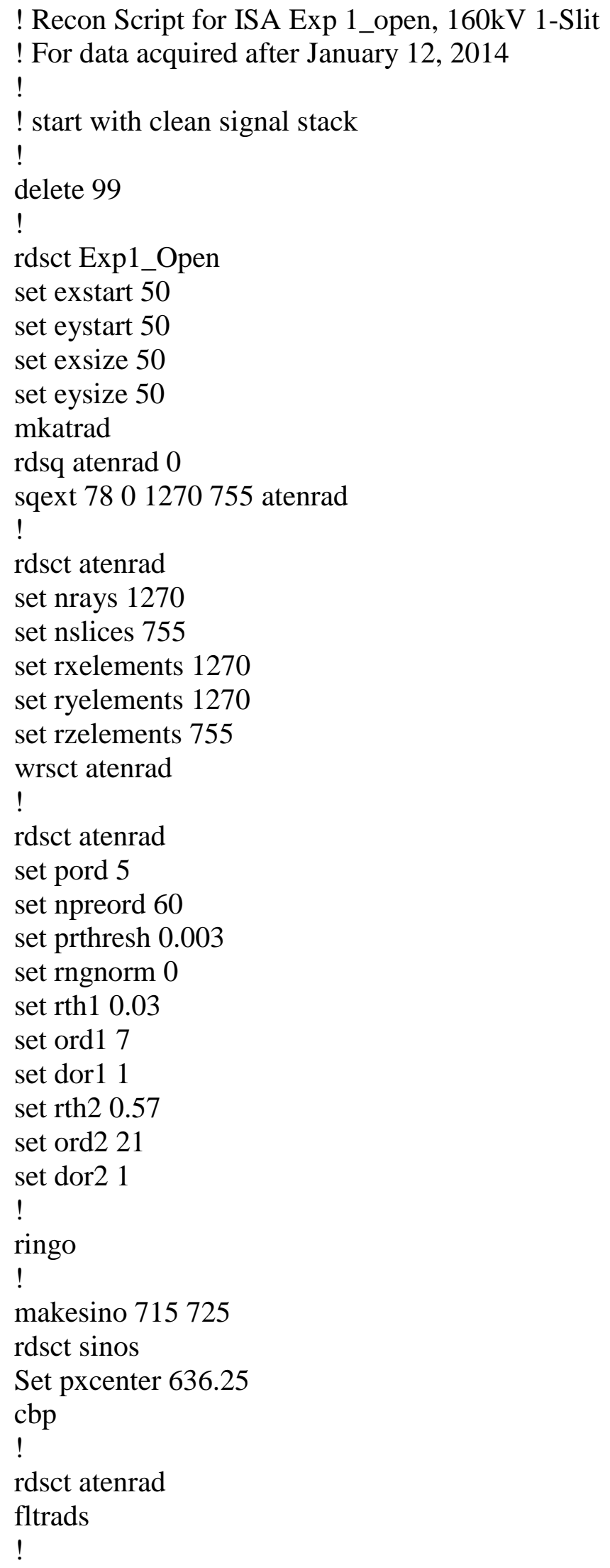


set ryelements 50
set rzoffset 0
set rzelements 400
set pxcenter 636.25
set pzcenter 532.0
set ryoffset 700
feldkamp
set ryoffset 750
feldkamp
set ryoffset 800
feldkamp
set ryoffset 850
feldkamp
set ryoffset 900
feldkamp
set ryoffset 950
feldkamp
set ryoffset 1000
feldkamp
rdsq recry 700
sqgty 0400 rz
!
! Reconstruction Complete 